OPEN ACCESS

Edited by: Michaela Kress, Innsbruck Medical University, Austria

Reviewed by: Stefania Ceruti, Università degli Studi di Milano, Italy Shuohao Sun, Johns Hopkins University, United States

*Correspondence: Guzel Sitdikova sitdikovaguze/@gmail.com

Received: 24 May 2017 Accepted: 14 July 2017 Published: 27 July 2017

Citation: Koroleva K, Mustafina A, Yakovlev A Hermann A, Giniatullin R and Sitdikova G (2017) Receptor Mechanisms Mediating the Pro-Nociceptive Action of Hydrogen Sulfide in Rat Trigeminal Neurons and Meningeal Afferents. Front. Cell. Neurosci. 11:226. doi: 10.3389/fncel.2017.00226

\section{Receptor Mechanisms Mediating the Pro-Nociceptive Action of Hydrogen Sulfide in Rat Trigeminal Neurons and Meningeal Afferents}

\author{
Kseniya Koroleva ${ }^{1}$, Alsu Mustafina ${ }^{1}$, Aleksey Yakovlev ${ }^{1}$, Anton Hermann ${ }^{2}$, \\ Rashid Giniatullin ${ }^{1,3}$ and Guzel Sitdikova ${ }^{1 *}$
}

\begin{abstract}
'Department of Human and Animal Physiology, Institute of Fundamental Medicine and Biology, Kazan Federal University, Kazan, Russia, ${ }^{2}$ Department of Cell Biology and Physiology, University of Salzburg, Salzburg, Austria, ${ }^{3}$ A.I. Virtanen Institute for Molecular Sciences, University of Eastern Finland, Kuopio, Finland
\end{abstract}

Hydrogen sulfide $\left(\mathrm{H}_{2} \mathrm{~S}\right)$, a well-established member of the gasotransmitter family, is involved in a variety of physiological functions, including pro-nociceptive action in the sensory system. Although several reports have shown that $\mathrm{H}_{2} \mathrm{~S}$ activates sensory neurons, the molecular targets of $\mathrm{H}_{2} \mathrm{~S}$ action in trigeminal (TG) nociception, implicated in migraine, remains controversial. In this study, using suction electrode recordings, we investigate the effect of the $\mathrm{H}_{2} \mathrm{~S}$ donor, sodium hydrosulfide (NaHS), on nociceptive firing in rat meningeal TG nerve fibers. The effect of NaHS was also explored with patch-clamp and calcium imaging techniques on isolated TG neurons. NaHS dramatically increased the nociceptive firing in TG nerve fibers. This effect was abolished by the TRPV1 inhibitor capsazepine but was partially prevented by the TRPA1 blocker HC 030031. In a fraction of isolated TG neurons, NaHS transiently increased amplitude of capsaicin-induced currents. Moreover, NaHS by itself induced inward currents in sensory neurons, which were abolished by the TRPV1 inhibitor capsazepine suggesting involvement of TRPV1 receptors. In contrast, the inhibitor of TRPA1 receptors HC 030031 did not prevent the NaHS-induced currents. Imaging of a large population of TG neurons revealed that NaHS induced calcium transients in $41 \%$ of tested neurons. Interestingly, this effect of $\mathrm{NaHS}$ in some neurons was inhibited by the TRPV1 antagonist capsazepine whereas in others it was sensitive to the TRPA1 blocker HC 030031. Our data suggest that both TRPV1 and TRPA1 receptors play a role in the pro-nociceptive action of NaHS in peripheral TG nerve endings in meninges and in somas of TG neurons. We propose that activation of TRPV1 and TRPA1 receptors by $\mathrm{H}_{2} \mathrm{~S}$ during neuro-inflammation conditions contributes to the nociceptive firing in primary afferents underlying migraine pain.

Keywords: pain, hydrogen sulfide, trigeminal nerve firing, trigeminal neurons, TRPV1-and TRPA1 receptors, $\mathrm{Ca}^{2+}$-imaging

Abbreviations: CBS, cystathionine beta-synthase; CGRP, calcitonin gene-related peptide; CSE, cystathionine gamma-lyase; $\mathrm{H}_{2} \mathrm{~S}$, hydrogen sulfide; NaHS, sodium hydrosulfide; TG, trigeminal. 


\section{INTRODUCTION}

Hydrogen sulfide $\left(\mathrm{H}_{2} \mathrm{~S}\right)$, a member of the gasotransmitter family along with nitric oxide (NO) and carbon monoxide, is involved in the regulation of great variety of physiological functions, including nociception and inflammation (Kawabata et al., 2007; Feng et al., 2013). The neuro-modulatory role of $\mathrm{H}_{2} \mathrm{~S}$ was shown in the central and peripheral nervous system where it promotes the induction of long-term potentiation (LTP) in hippocampus (Abe and Kimura, 1996), inhibits giant depolarizing potentials in neonatal hippocampus (Yakovlev et al., 2017), affects NMDA-mediated currents (Abe and Kimura, 1996; Yakovlev et al., 2017), increases the transmitter release from motor nerve endings (Sitdikova et al., 2011; Gerasimova et al., 2013, 2015), or initiates contractile responses of the rat urinary bladder by stimulation of primary afferent neurons (Patacchini et al., 2004).

Increasing evidence suggests $\mathrm{H}_{2} \mathrm{~S}$ to play a role in the emergence and conductance of somatic and visceral pain (Okubo et al., 2012). Intracolonical administration of sodium hydrosulfide (NaHS), a $\mathrm{H}_{2} \mathrm{~S}$ donor, induced nociceptive behavior with abdominal hyperalgesia/allodynia (Matsunami et al., 2009). NaHS produced mechanical hyperalgesia in the rat hind paw in response to intraplantar administration (Kawabata et al., 2007). On the other hand, NaHS activates ATP-dependent $\mathrm{K}^{+}$channels in different tissues (Tang et al., 2005; Mustafina et al., 2015) which may underlie the antinociceptive effects of NaHS (Distrutti et al., 2006).

Endogenously $\mathrm{H}_{2} \mathrm{~S}$ is produced from L-cysteine by the enzymes cystathionine $\beta$-synthase (CBS), cystathionine $\gamma$-lyase (CSE) and 3-mercaptopyruvate sulfurtransferase along with additional contribution of cysteine aminotransferase or D-amino acid oxidase (Abe and Kimura, 1996; Renga, 2011). It was shown that CBS is widely expressed in rat trigeminal (TG) neurons (Feng et al., 2013) and its expression is upregulated in response to inflammatory pain with subsequent increase of the excitability of TG neurons by suppression of $\mathrm{K}^{+}$conductance (Miao et al., 2014). It was reported that CBS is colocalized with transient receptor potential, vanilloid 1 (TRPV1) receptors in colon specific dorsal root ganglion (DRG) neurons (Xu et al., 2009; Qu et al., 2013). TRPV1 receptors undergo sensitization in response to inflammation which is mediated by the increased expression of CBS on DRG (Zhu et al., 2015).

Several recent publications indicated the ability of $\mathrm{H}_{2} \mathrm{~S}$ to activate TRPV1 or TRPA1 receptors in vitro and in vivo experiments. Thus, the TRPV1 antagonist prevented NaHS-evoked luminal chloride secretion (Storti et al., 2015). NaHS-induced constriction of smooth muscle cells of airways and $\mathrm{H}_{2} \mathrm{~S}$-evoked intestinal motility were abolished by the TRPV1 antagonists (Trevisani et al., 2005; Bhatia et al., 2006). Moreover, NaHS increased the afferent neuronal activity in gut and induced inward currents in DRG neurons which were inhibited by TRPV1 antagonists (Lu et al., 2014).

However, there is also evidence indicating activation of TRPA1 receptors by $\mathrm{H}_{2} \mathrm{~S}$. Activation of capsaicin-sensitive sensory nerves through TRPA1 receptors by NaHS-induced vasodilatation resulting from the release of the vasoactive neuropeptides calcitonin gene-related peptide (CGRP) and substance P (Pozsgai et al., 2012; Hajna et al., 2016). Indirect evidence shows that activation of TRPA1 channels by $\mathrm{H}_{2} \mathrm{~S}$ resulted in mechanical hyperalgesia and allodynia in mice (Okubo et al., 2012) whereas TRPA1 did not participate in pro-nociceptive effects of $\mathrm{H}_{2} \mathrm{~S}$ in visceral tissues (Andersson et al., 2012). There is abundance evidence that $\mathrm{H}_{2} \mathrm{~S}$ affects TRP channels in sensory neurons, but the molecular target of $\mathrm{H}_{2} \mathrm{~S}$ action in nociceptive system remains to be determined.

The aim of this study was to explore the role of TRP receptors in the firing of TG nerve fibers induced by NaHS using extracellular recordings of peripheral branches of the TG nerve in isolated rat meninges and patch clamp recordings of TRPV1 currents as well as $\mathrm{Ca}^{2+}$-imaging of rat TG neurons.

\section{MATERIALS AND METHODS}

\section{Preparation and Solutions}

All animal experiments were performed in accordance with the European Community Council Directive of September 22, 2010 (2010/63/EEC) and approved by the Animal Care and Use Committee of the University of Eastern Finland and the Ethics Committee of Kazan Federal University. Electrical activity of TG nerve was recorded using isolated hemiskull preparations obtained from adult (P35-36) rats as described previously (Shatillo et al., 2013). Firing activity was recorded from the nervus spinosus (V3 branch of the TG nerve) which was isolated and cleaned from the dura mater. This nerve innervates a region of the medial meningeal artery, supposed to initiate migraine pain and this model is widely used to investigate molecular mechanisms of migraine pain. The isolated preparation was washed for $20 \mathrm{~min}$ with Krebs solution containing (in $\mathrm{mM}$ ): $120 \mathrm{NaCl} ; 2.5 \mathrm{KCl} ; 2 \mathrm{CaCl}_{2} ; 1 \mathrm{MgCl}_{2} ; 11$ glucose; $1 \mathrm{NaHPO}_{4}$; $24 \mathrm{NaHCO}_{3}$ constantly gassed with $95 \% \mathrm{O}_{2} / 5 \% \mathrm{CO}_{2}$ and the $\mathrm{pH}$ kept at 7.2-7.4.

TG neurons were isolated from P9-P12 rats. Animals were anesthetized and decapitated. TG ganglia were excised and enzymatically dissociated in F12 medium containing $0.25 \mathrm{mg} / \mathrm{ml}$ trypsin, $1 \mathrm{mg} / \mathrm{ml}$ collagenase, and $0.2 \mathrm{mg} / \mathrm{ml}$ DNAase (Sigma) at $37^{\circ} \mathrm{C}$. Cells were plated on poly-l-lysine-coated glasses in F12 medium with $10 \%$ fetal bovine serum and cultured for $1-2$ days at $37^{\circ} \mathrm{C}$ in an atmosphere containing $5 \% \mathrm{CO}_{2}$. During experiments cells were continuously perfused (at $2 \mathrm{ml} / \mathrm{min}$ ) with a solution containing (in $\mathrm{mM}$ ): $148 \mathrm{NaCl} ; 5 \mathrm{KCl} ; 1 \mathrm{MgCl}_{2}$; $2 \mathrm{CaCl}_{2} ; 10$ HEPES; 10 D-Glucose; $\mathrm{pH}$ adjusted to 7.2 with $\mathrm{NaOH}$. The intracellular solution for patch clamp experiments contained (in mM): $145 \mathrm{KCl} ; 2 \mathrm{MgCl}_{2} ; 10$ HEPES; 5 EGTA; $0.5 \mathrm{CaCl}_{2} ; 2 \mathrm{Mg}$-ATP; $0.5 \mathrm{Na}-\mathrm{GTP} ; 5 \mathrm{KCl} ; \mathrm{pH}$ adjusted to 7.2 with $\mathrm{KOH}$.

For $\mathrm{Ca}^{2+}$ imaging experiments cells were incubated for $40 \mathrm{~min}$ at $37^{\circ} \mathrm{C}$ in F12 medium supplemented with FBS $10 \%$ (Gibco Invitrogen, Carlsbad, CA, USA) containing fluo-3-AM ( $5 \mu \mathrm{M}$, Life Technologies, Foster City, CA, USA) followed by a 10-15 min washout period. 


\section{Chemicals}

Capsaicin, HC 030031 and capsazepine were dissolved in dimethyl sulfoxide (DMSO), dithiothreitol (DTT) - in external solution. DMSO in used concentration did not change the nociceptive activity (Zakharov et al., 2015). All substances were purchased from Sigma-Aldrich (St. Louis, MO, USA). NaHS (Sigma-Aldrich, St. Louis, MO, USA) was used as a source of $\mathrm{H}_{2} \mathrm{~S}$. In solution $\mathrm{NaHS}$ dissociates to give $\mathrm{HS}^{-}$which associates with $\mathrm{H}^{+}$to produce $\mathrm{H}_{2} \mathrm{~S}$. At $20^{\circ} \mathrm{C}-22.3 \%$ of total sulfide is present as $\mathrm{H}_{2} \mathrm{~S}$ (Sitdikova et al., 2014). The real-time measurements of $\mathrm{H}_{2} \mathrm{~S}$ in the chamber using amperometric sensors indicate a rapid loss of sulfide via $\mathrm{H}_{2} \mathrm{~S}$ volatilization by bubbling with about $50 \% \mathrm{H}_{2} \mathrm{~S}$ loss within 3 min (Deleon et al., 2012; Sitdikova et al., 2014). In our experiments NaHS was used in a concentration of $100 \mu \mathrm{M}$ which yields about $11 \mu \mathrm{M} \mathrm{H} \mathrm{H}_{2} \mathrm{~S}$ in the perfusion system which constantly flows to the recording chamber. Stock solutions of NaHS were prepared immediately before each experiment and kept hermetically sealed in a dark place.

\section{Electrophysiology}

TG nerve firing was recorded using a DAM 80 amplifier (band pass $0.001-3 \mathrm{kHz}$, gain 1000; World Precision Instruments, Sarasota, FL, USA). The nervus spinosus was placed inside the fire-polished glass recording microelectrode with a tip diameter of $\sim 150 \mu \mathrm{m}$, filled with Krebs solution. A recovery period of at least $15 \mathrm{~min}$ was used to obtain stable baseline conditions. Control recordings of meningeal spikes were performed for $10 \mathrm{~min}$ previous to drug application. Signals were digitized at $125 \mathrm{kHz}$ using a data acquisition board NI PCI6221 (National Instruments, Austin, TX, USA), and WinEDR software (Strathclyde University, Glasgow, UK). Five standard deviations (SD) were used to set the threshold for spike detection.

TRPV1 receptors are predominantly expressed in smalland medium-diameter neurons, which were used in our patch clamp experiments. TRPV1 currents were recorded at a holding potential of $-70 \mathrm{mV}$ using the whole-cell configuration of the patch clamp technique. TRPV1 currents were evoked by local application of capsaicin in a concentration of $1 \mu \mathrm{M}$ for $2 \mathrm{~s}$ using a fast perfusion system (Rapid Solution Changer, RSC-200, BioLogic Science Instruments, Grenoble, France), with a solution exchange rate of $\sim 20 \mathrm{~ms}$. To prevent the desensitization of TRPV1 receptors, agonist was applied at intervals of $5 \mathrm{~min}$. Responses to capsaicin were measured using a HEKA-10 amplifier and HEKA Patch Master Software (HEKA Electronic, Germany).

\section{Calcium Imaging}

Fluorescence signals of neurons were recorded by microscope imaging setup (TILL Photonics GmbH, Munich, Germany) with a light excitation wavelength of $488 \mathrm{~nm}$ using respective filters. Images were collected in a time-lapse mode $(500 \mathrm{~ms}$ exposure time) with a 12-bit CCD camera (SensiCam, Kelheim, Germany). Fluorescent signals from single cells were quantified as $\triangle F / F 0$, where $F 0$ is the background subtracted baseline fluorescence and $\Delta F$ is the increment over baseline. The inhibitor of TRPV 1 -capsazepine $(10 \mu \mathrm{M})$, the inhibitor of TRPA1-HC $030031(50 \mu \mathrm{M})$, NaHS $(100 \mu \mathrm{M}, 2 \mathrm{~s})$ and capsaicin $(1 \mu \mathrm{M}$, 2 s) were applied via a fast perfusion system as indicated above, followed by application of a $50 \mathrm{mM} \mathrm{KCl-containing}$ solution to differentiate neurons. Data were analyzed off-line using Origin Pro 2015 (MicroCal, Northampton, MA, USA) software.

\section{Statistical Analysis}

For each experiment we used at least three independent replicates (animals) and $n$ means the number of cells. Normality of sample data was evaluated with Shapiro-Wilk test and for equal variances using F-test Origin Pro 2015 (OriginLab Corp., Northampton, MA, USA). Differences were considered as statistically significant at $p<0.05$. All values are given as mean \pm SEM. Statistical significance was determined by paired Student's $t$-test and Mann-Whitney test.

\section{RESULTS}

\section{NaHS Increases Firing in Meningeal Nerve Terminals}

First, we tested if $\mathrm{H}_{2} \mathrm{~S}$ can induce the nociceptive effect in meningeal nerves using a technique of suction electrode recording from meningeal TG nerve fibers (Zakharov et al., 2015). The peripheral part of the TG nerve (nervus spinosus) was placed inside the microelectrode and the orthodromic spontaneously generated action potentials (AP; spikes) generated at the periphery were recorded (Figure 1). Bath application of $100 \mu \mathrm{M}$ NaHS induced a significant increase in the frequency of nociceptive spikes during the first $5 \mathrm{~min}$ to $320 \pm 88 \%$ of control (control $0.53 \pm 0.08 \mathrm{~s}^{-1}$, vs. $1.35 \pm 0.13 \mathrm{~s}^{-1}$ in the presence of NaHS; $n=10, p<0.0001$; Figures 1A,D). During the next 5 min of application, the frequency of spikes did not differ significantly from control $\left(0.95 \pm 0.30 \mathrm{~s}^{-1}, p=0.2\right)$ and it returned to control level after $15 \mathrm{~min}$ of drug application $\left(0.40 \pm 0.01 \mathrm{~s}^{-1} ; p=0.3\right.$; Figure 1A).

As previous studies suggested the action of NaHS in different tissues could be mediated either by TRPV1 or TRPA1 receptors (Trevisani et al., 2005; Andersson et al., 2012; Okubo et al., 2012; Lu et al., 2014; Hajna et al., 2016), we next examined the pro-nociceptive effect of NaHS in the presence of the specific inhibitors of these receptors. Inhibition of TRPV1 receptors by capsazepine $(25 \mu \mathrm{M})$ did not significantly change TG nerve firing $\left(0.40 \pm 0.11 \mathrm{~s}^{-1}\right.$ in control, vs. $0.32 \pm 0.13 \mathrm{~s}^{-1}$ in capsazepine; $n=6, p=0.14$ ). Remarkably, the subsequent application of NaHS in the presence of capsazepine did not affect the frequency of nociceptive firing. The frequency of AP was $0.56 \pm 0.30 \mathrm{~s}^{-1}(p=0.5)$ during first $5 \mathrm{~min}$ of NaHS application, $0.44 \pm 0.27 \mathrm{~s}^{-1}(p=0.85)$ and $0.27 \pm 0.15 \mathrm{~s}^{-1}$ during 10 and $15 \mathrm{~min}$ of application, respectively $(p=0.25$; Figures 1B,D). The inhibition of TRPA1 by HC 030031 $(50 \mu \mathrm{M})$ did not change firing itself $\left(0.61 \pm 0.11 \mathrm{~s}^{-1}\right.$ in control, vs. $0.55 \pm 0.09 \mathrm{~s}^{-1}$ in HC 030031; $n=7, p=0.51$ ), however, partially prevented the action of NaHS (increase to $161 \pm 13 \% ; 0.85 \pm 0.13 \mathrm{~s}^{-1} ; n=7, p=0.007$; Figures 1C,D). 

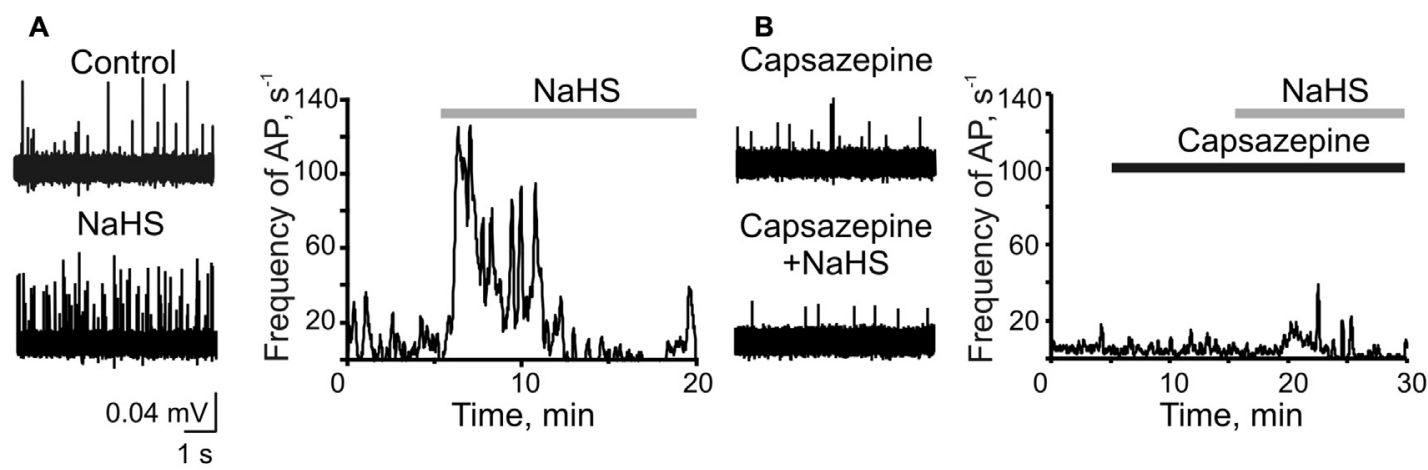

C

HC 030031
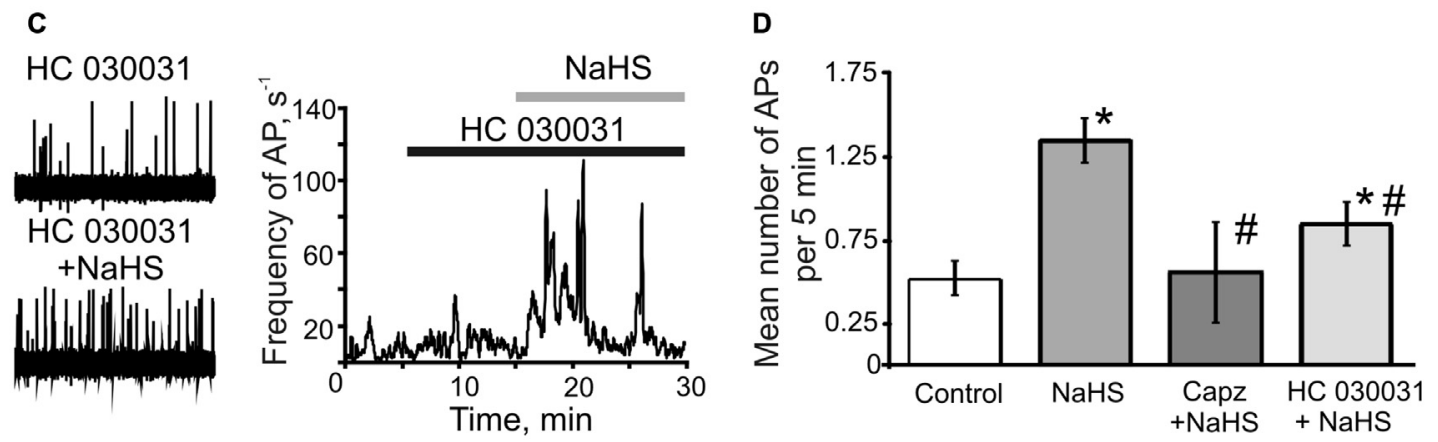

FIGURE 1 | The role of TRPV1 and TRPA1 receptors in the effects of sodium hydrosulfide (NaHS) on trigeminal (TG) nerve firing. (A) Representative traces of TG nerve firing in meningeal hemiskull preparation (left panel) and frequency plots of action potentials (AP) vs. time during single experiments (right panel) in control and after application of NaHS (100 $\mu$ M); (B) after inhibition of TRPV1 receptors by capsazepine (10 $\mu$ M) and capsazepine + NaHS (C) after inhibition of TRPA1 receptors by HC $030031(50 \mu \mathrm{M})$ and $\mathrm{HC} 030031$ + NaHS. The solid bars indicate the application time of NaHS, capsazepine and HC 030031. (D) Plot of mean number of AP per 5 min during control, after application of NaHS, capsazepine (capz) + NaHS and HC $030031+\mathrm{NaHS}$. ${ }^{*} p<0.05$ compared to control; ${ }^{*} p<0.05$ compared to the effect of $\mathrm{NaHS}$.

The NaHS effect after inhibition of TRPA1 was significantly lower than in control conditions. During 10 and $15 \mathrm{~min}$ of NaHS application the frequency of AP returned to the control level $\left(0.70 \pm 0.12 \mathrm{~s}^{-1}, p=0.13\right.$ and $0.82 \pm 0.19 \mathrm{~s}^{-1}$, $p=0.27$, respectively; Figure $1 \mathrm{C}$ ). These results suggested the involvement of TRPV1 and TRPA1 receptors in the pro-nociceptive firing induced by NaHS in TG nerve fibers in meninges.

\section{NaHS Induces TRPV1 Mediated Currents in Isolated TG Neurons}

Next, we studied the effect of NaHS on TRPV1 receptors in single TG neurons using patch clamp recordings. The application of the TRPV1 receptor agonist capsaicin $(1 \mu \mathrm{M}, 2 \mathrm{~s})$ induced inward currents in 22 out of 31 neurons with an average amplitude of $1345 \pm 330 \mathrm{pA}$ ( $n=22$; Figures 2A,B). In control, the repetitive application of capsaicin (interval $5 \mathrm{~min}$ ) revealed two fractions of neurons: in one fraction, the amplitude of TRPV1 currents did not significantly change ( $n=14$, Figure 2C), whereas in another fraction rundown of currents was observed ( $n=8$, Figure 2D). These findings are consistent with an intrinsic heterogeneity of TRPV1 receptors with different rates of desensitization which was found in isolated sensory neurons previously (Akopian et al., 2007; Storti et al., 2015). We did not reveal differences in the membrane capacitance between two groups of TG neurons $(36.9 \pm 4.7 \mathrm{pF}, n=14$ vs. $33.4 \pm 7.7 \mathrm{pF}$, $n=8, p=0.67)$. Superfusion with NaHS $(100 \mu \mathrm{M})$ for 5 min revealed bidirectional effects on the fractions of cells with a different rate of desensitization. In a fraction of 14 cells with a low rate of desensitization, NaHS transiently increased currents from $1578 \pm 501 \mathrm{pA}$ to $2357 \pm 715 \mathrm{pA}(n=14$, $p=0.0065$ ) followed by rundown during 10 and $15 \mathrm{~min}$ of NaHS application (Figures 2A,C). In other eight cells, we only observed a reduction of capsaicin-induced currents after $5 \mathrm{~min}$ exposure to NaHS from control value of $768 \pm 168 \mathrm{pA}$ to $414 \pm 141 \mathrm{pA}$ in the presence of NaHS $(n=8, p=0.016$; Figures 2B,D). In both cases, the effect of NaHS was not washable. The activating NaHS effects on TRPV1 currents could be explained by its reducing action on disulfide bonds of the TRPV1 channel protein (Susankova et al., 2006). Indeed, pre-application of $1 \mathrm{mM}$ DTT prevented the increase of the current amplitude by NaHS. Thus, in the presence of DTT, NaHS decreased the amplitude of TRPV1 currents from $1360 \pm 330 \mathrm{pA}$ to $1025 \pm 371 \mathrm{pA}(n=11, p=0.0033)$ by $5 \mathrm{~min}$ of application with further reduction to $622 \pm 171(p<0.0002)$ and then to $532 \pm 167 \mathrm{pA}(p<0.0001)$, by 10 and $15 \mathrm{~min}$, respectively (data not shown).

To investigate the direct effect of NaHS on TRPV1 currents, NaHS $(100 \mu \mathrm{M})$ was applied through the fast perfusion system for 2 s. In cells responding to capsaicin, NaHS induced an 



FIGURE 2 | Facilitatory and inhibitory effects of NaHS on capsaicin induced currents in rat TG neurons. (A,B) Representative traces of capsaicin evoked currents (Caps, $1 \mu \mathrm{M}, 2 \mathrm{~s}$, short bars above traces) in control and during bath application of $100 \mu \mathrm{M}$ NaHS for 15 min (solid bar above traces). Capsaicin was applied at an interval of 5 min to prevent desensitization of TRPV1 receptors. (C,D) Average amplitude of TRPV1 currents in control (three subsequent capsaicin application) and during NaHS application (solid bars above traces). Notice the increase of mean amplitude of TRPV1 currents after 5 min of NaHS perfusion in (C) and the constant decrease of capsaicin evoked currents in (D). (E) Representative traces of currents evoked by focal application of $1 \mu \mathrm{M}$ capsaicin (Caps, $2 \mathrm{~s}$ ) and $100 \mu \mathrm{M}$ NaHS ( $2 \mathrm{~s})$ in control and after inhibition of TRPV1 receptors by capsazepine (10 $\mu \mathrm{M})$; after washout and after inhibition of TRPA1 receptors by HC 030031 (50 $\mu$ M). Notice that $\mathrm{NaHS}$ and capsaicin evoked currents were completely abolished by capsazepine. ${ }^{*} p<0.05$ compared to the third application of capsaicin in control.

inward current with an average amplitude of $413 \pm 114 \mathrm{pA}$ $(n=12$; Figure 2E). Application of the TRPV1 receptor antagonist capsazepine $(10 \mu \mathrm{M})$ completely blocked currents induced by NaHS and capsaicin ( $n=5$, Figure 2E). NaHS and capsaicin-induced currents were washable from the capsazepine inhibition. Subsequent application of the TRPA1 receptor selective antagonist HC $030031(50 \mu \mathrm{M})$ on the same cell did not affect currents induced by NaHS and capsaicin $(n=5$, Figure 2E). These data suggest that $\mathrm{NaHS}$ can directly activate TRPV1 receptors in rat TG neurons.

\section{NaHS Increases the Intracellular $\mathrm{Ca}^{2+}$ Concentration in Isolated TG Neurons}

In order to explore the action of the $\mathrm{H}_{2} \mathrm{~S}$ donor on intracellular $\mathrm{Ca}^{2+}$ level in a large population of isolated neurons, we applied capsaicin $(1 \mu \mathrm{M})$ and $\mathrm{NaHS}(100 \mu \mathrm{M})$ for $2 \mathrm{~s}$ before and after inhibition of TRPV1 or TRPA1 receptors by capsazepine $(10 \mu \mathrm{M})$ or HC $030031(50 \mu \mathrm{M})$, respectively. The subsequent application of $\mathrm{KCl}(50 \mathrm{mM})$ was used to distinguish neurons from glial cells (Kilic et al., 2017). As shown in Figures 3A,B NaHS induced $\mathrm{Ca}^{2+}$ transients in $41 \%$ neurons (104 of 251 cells); $43 \%$ of neurons tested were also sensitive to capsaicin (107 cells; Figure 3B). In 59\% of cells responding to capsaicin, NaHS also induced an increase of intracellular $\mathrm{Ca}^{2+}$ (63 of 107 cells). Inhibition of TRPV1 receptors by capsazepine abolished $\mathrm{Ca}^{2+}$ responses evoked by NaHS in $37 \%$ of cells, which responded to NaHS and in $63 \%$ of cells the increase of $\left[\mathrm{Ca}^{2+}\right]_{\text {in }}$ evoked by NaHS was still observed (Figures 3A,C). After inhibition of TRPA1 receptors by HC $030031,80 \%$ of cells still showed an increase of $\left[\mathrm{Ca}^{2+}\right]_{\text {in }}$ by NaHS application and in 20\% of cells the NaHS response was eliminated (Figures 3A,C). In summary, in this approach 
the action of NaHS was partially sensitive to TRPV1 and TRPA1 antagonists.

\section{DISCUSSION}

The main finding of our study indicates that $\mathrm{H}_{2} \mathrm{~S}$ induces an increase of firing activity in rat TG nerve and this effect is mediated by activation of both TRPV1 and TRPA1 receptors. In somas of TG neurons NaHS caused two types of effects on capsaicin evoked currents. In a fraction of neurons, NaHS induced a transient initial increase of the current amplitude followed by a subsequent decrease of responses. In the other fraction of cells, NaHS induced a progressive decline of TRPV1 currents. Moreover, $\mathrm{H}_{2} \mathrm{~S}$ when locally applied to TG neurons elicits inward currents which were inhibited by the TRPV1 antagonist capsazepine but was not sensitive to the inhibitor of the TRPA1 receptors HC 030031. Furthermore, NaHS generated $\mathrm{Ca}^{2+}$ transients in TG neurons which were prevented by the inhibitors of TRPV1 and TRPA1 receptors. We propose that both TRPV1 and TRPA1 receptors in peripheral nerve endings in meninges and in somas of sensory neurons are involved in the pro-nociceptive action of $\mathrm{H}_{2} \mathrm{~S}$ in the trigeminovascular system.

\section{$\mathrm{H}_{2} \mathrm{~S}$ Increases Firing in TG Nerve by Activation of TRPV1 and TRPA1 Receptors}

The TG system is directly involved in sensory and nociceptive conductance and TG nerve firing is involved in pain initiation during migraine. TRPA1 and TRPV1 receptors are widely expressed in capsaicin-sensitive sensory nerves (Huang et al., 2012). Activation of TRPV1 and TRPA1 on meningeal nerve endings induces the release of vasoactive neuropeptides CGRP and substance $\mathrm{P}$ and contributes to different forms of headache including migraine (Giniatullin et al., 2008; Benemei et al., 2013). Our results demonstrate that the donor of $\mathrm{H}_{2} \mathrm{~S}-\mathrm{NaHS}$ directly increases firing in TG nerve and this effect is mainly mediated by activation of TRPV1 as
A neuron 1
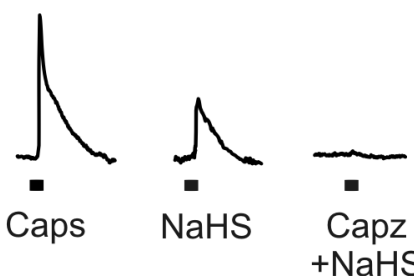

neuron 2

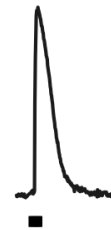

Caps

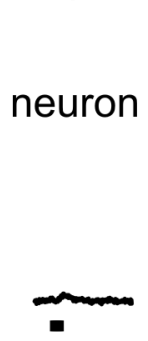

Caps

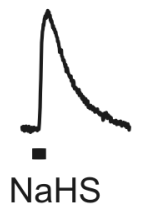

$\mathrm{NaHS}$



$+\mathrm{NaHS}$

$\overbrace{\mathrm{KCl}}^{1}$

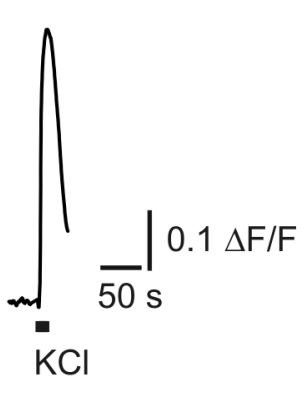

B
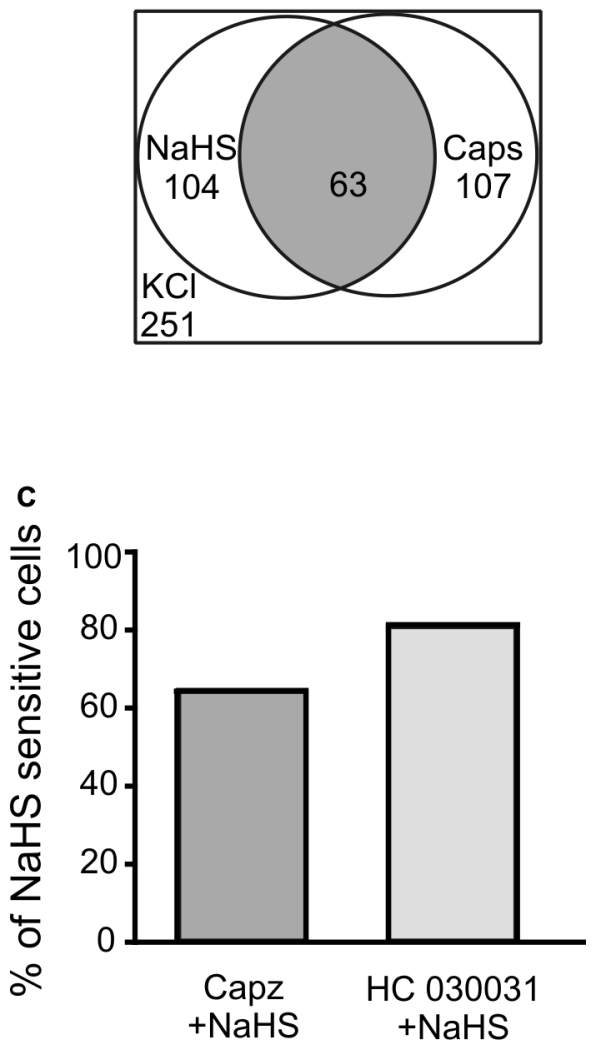

FIGURE 3 | NaHS evokes calcium transients in TG neurons. (A) Representative traces recorded from cultured TG neurons. NaHS (100 $\mu$ M) and capsaicin (Caps, $1 \mu \mathrm{M}$ ) were applied for $2 \mathrm{~s}$ to TG ganglion cells loaded with fluo 3-AM $(5 \mu \mathrm{M})$. Neurons were distinguished from other cell types due to Ca ${ }^{2+}$ responses induced by potassium depolarization ( $50 \mathrm{mM} \mathrm{KCl}$ for $2 \mathrm{~s}$ ). In neuron $1 \mathrm{TRPV} 1$ inhibitor capsazepine (capz, $10 \mu \mathrm{M})$ abolished NaHS induced Ca $2+$ transient; in neuron 2 capsazepine did not affect NaHS induced $\mathrm{Ca}^{2+}$ transient; in neuron 3 TRPA1 inhibitor HC 030031 (50 $\mu$ M) abolished NaHS evoked response. (B) Graph indicating populations of capsaicin and NaHS sensitive TG neurons and their overlap. In a total of 251 imaged neurons (selected by their response to KCl) 104 (41\%) were activated by NaHS $(100 \mu \mathrm{M})$ and $107(43 \%)$ by capsaicin $(1 \mu \mathrm{M})$. In $59 \%$ of capsaicin sensitive cells NaHS also induced an increase of intracellular Ca ${ }^{2+}(63$ cells from 107, gray area). (C) Percentage of cells responding to NaHS (100\%) after inhibition of TRPV1 (Capz + NaHS) and TRPA1 (HC $030031+\mathrm{NaHS})$ receptors. 
capsazepine, TRPV1 antagonist, completely abolished the effect of NaHS. At the same time the TRPA1 antagonist HC 030031 partially prevented the increase of TG nerve firing indicating the involvement of TRPA1 receptors in the $\mathrm{H}_{2} \mathrm{~S}$ effect.

A number of indirect studies indicate the activation of TRPV1 and TRPA1 in afferent endings by $\mathrm{H}_{2} \mathrm{~S}$. It was shown that NaHS similar to capsaicin induced the release of CGRP and substance $\mathrm{P}$ from sensory nerves in the airways of guinea pig which causes bronchoconstriction in vivo (Patacchini et al., 2004; Trevisani et al., 2005). NaHS evoked contractions of the urinary bladder by activation of capsaicin-sensitive afferents and release of sensory neuropeptides (Patacchini et al., 2004). In the guinea-pig and human colon $\mathrm{H}_{2} \mathrm{~S}$ caused mucosal $\mathrm{Cl}^{-}$secretion (Schicho et al., 2006) and increased afferent firing in rat intestinal mesenteric nerves by activation of TRPV1 in afferent endings ( $\mathrm{Lu}$ et al., 2014). On the other hand activation of TRPA1 by NaHS in afferent nerve fibers was reported to mediate an increased cutaneous blood flow by the release of CGRP and substance P in the mouse ear model (Hajna et al., 2016), whereas vasodilatory effects of $\mathrm{H}_{2} \mathrm{~S}$ were reduced in mice lacking TRPA1 receptors (Pozsgai et al., 2012).

\section{$\mathrm{H}_{2} \mathrm{~S}$ Directly Activates TRPV1 Receptors and Increases Intracellular $\mathrm{Ca}^{2+}$ Concentrations in Isolated TG Neurons}

The focal application of NaHS on TG neurons induced inward currents similar to capsaicin which were inhibited by the TRPV1 antagonist capsazepine but were not sensitive to the inhibitor of TRPA1 antagonist HC 030031, which indicates a direct activation of TRPV1 receptors by $\mathrm{H}_{2} \mathrm{~S}$. At the same time superfusion of TG neurons with NaHS induced a bidirectional effect on capsaicin induced currents. In $63 \%$ of neurons NaHS induced an increase of the current amplitude during first minutes with subsequent desensitization, which can be explained by the reduction of disulfide bonds by $\mathrm{H}_{2} \mathrm{~S}$. Indeed, the sulfhydryl redox agent DTT in our experiments prevented the facilitating effect of NaHS. The redox modulation of TRPV1 receptors is well-known and it was shown than DTT greatly potentiated both native and recombinant rat TRPV1 channels at extracellularly located sites (Susankova et al., 2006). $\mathrm{H}_{2} \mathrm{~S}$ is known for its reducing action which is responsible for its effects on $\mathrm{Ca}^{2+}$-activated $\mathrm{K}^{+}$channels and NMDA-receptors (Abe and Kimura, 1996; Sitdikova et al., 2010; Kimura, 2016). In 36\% of cells a constant decrease of TRPV1 currents was observed during NaHS superfusion, which may be explained by the rapid desensitization of TRPV1 receptors. This suggestion is supported by the variability in desensitization of capsaicin responses between different cell fractions. Indeed, the existence of several pools of TRPV1 receptors with slow and fast kinetics and with distinct rates of desensitization have been reported in TG neurons (Akopian et al., 2007; Storti et al., 2015; Zakharov et al., 2015) which may be determined by their lipid environment, coupling to caveolin, functional states, including redox state or phosphorylation (Storti et al., 2015). Moreover, the kinetics of TRPV1-mediated current depends on the co-expression of TRPA1 channels, with likely involvement of intracellular $\mathrm{Ca}^{2+}$ or other intracellular messengers affecting TRPV1 receptor desensitization (Masuoka et al., 2017). Similarly to our findings, in DRG neurons NaHS directly induced inward currents which were inhibited by capsazepine and A784168 (Lu et al., 2014). Our results suggest that $\mathrm{H}_{2} \mathrm{~S}$ is a putative agonist of TRPV1 receptors in somas of TG neurons.

Fluorescence studies demonstrate that $\mathrm{H}_{2} \mathrm{~S}$ increased the intracellular $\mathrm{Ca}^{2+}$ level in $41 \%$ of TG neurons cells, however, only $59 \%$ of $\mathrm{H}_{2} \mathrm{~S}$-sensitive cells responded to capsaicin, which reflects variability of $\mathrm{H}_{2} \mathrm{~S}$ molecular targets and co-expression of other types of calcium-permeable receptors in TG neurons. Thus, in DRG neurons, the TRPV1 current densities were significantly smaller in allyl isothiocyanate (AITC)-sensitive DRG neurons than in AITC-insensitive cells and spontaneous TRPA1 channel activity inhibited the TRPV1 channels via $\mathrm{Ca}^{2+}$ elevation (Masuoka et al., 2017). Indeed $37 \%$ of $\mathrm{H}_{2} \mathrm{~S}$ induced $\mathrm{Ca}^{2+}$ transients were inhibited by capsazepine which indicates mainly activation of TRPV1 receptors. However, $20 \%$ of $\mathrm{H}_{2} \mathrm{~S}$ induced $\mathrm{Ca}^{2+}$ transients were also abolished by HC 030031 indicating the activation of TRPA1 receptors in a small fraction of neurons, which was supported by a number of other studies. It was shown that in TG neurons application of NaHS increased $\left[\mathrm{Ca}^{2+}\right]_{\text {in }}$ in $20-42 \%$ of capsaicin-sensitive neurons with close correspondence between neurons that responded to NaHS and to AITC (Hajna et al., 2016). NaHS also evoked inward currents in DRG neurons and in CHO cells expressing TRPA1 receptors, which were inhibited by a TRPA1 antagonist (Miyamoto et al., 2011; Andersson et al., 2012; Ogawa et al., 2012).

However, it should be noted that high concentrations of NaHS (1-10 mM) were used in those studies which may activate TRPA1 indirectly by formation of reactive oxygen species (Andersson et al., 2008). Moreover, the main mechanism of TRPA1 activation appears to be oxidation of reactive cysteine residues whereas reducing agents induce an inhibition of TRPA1 (Macpherson et al., 2007). $\mathrm{H}_{2} \mathrm{~S}$ being a reducing agent cannot induce direct oxidation of the thiol groups of proteins (Greiner et al., 2013). Recent studies report that polysulfides generated in $\mathrm{NaHS}$ and $\mathrm{Na}_{2} \mathrm{~S}$ solutions or by the chemical interaction of $\mathrm{H}_{2} \mathrm{~S}$ and $\mathrm{NO}$ are able to activate TRPA1 receptors (Hatakeyama et al., 2015; Kimura, 2016; Miyamoto et al., 2017). It appears possible therefore that the effective molecules which activate TRPA1 receptors in our and previous studies were polysulfides. However, in our experiments relatively low concentration of NaHS (100 $\mu \mathrm{M}$, effectively $11 \mu \mathrm{M}$, see "Materials and Methods" Section) were used which probably is insufficient to generate sufficient amounts of polysulfides to activate TRPA1. Moreover, TRPV 1 stimulation by $\mathrm{H}_{2} \mathrm{~S}$ can cause $\mathrm{Ca}^{2+}$ dependent desensitization of TRPA1 receptor as TRPA1 is highly co-expressed with TRPV1 (Akopian et al., 2007; Palazzo et al., 2013).

The gating machinery of the TRPV1 receptor is complicated as different ligands are acting at distinct extra- and intracellular sites. The sites responsible for the reducing action of DTT are 
located at the extracellular part of the TRPV1 protein (Susankova et al., 2006) whereas capsaicin acts from the intracellular side (Gavva et al., 2004). Thus, the S512Y and Y511A point mutations at the intracellular part of the S3 segment were able to eliminate capsaicin sensitivity (Jordt and Julius, 2002). As the effect of NaHS was inhibited by capsazepine, a competitive antagonist of the TRPV1 receptor with structural similarities to capsaicin (Pingle et al., 2007), we propose that $\mathrm{H}_{2} \mathrm{~S}$ activates the gating of the TRPV1 receptor at the same locus as capsaicin. However, the exact mechanisms of $\mathrm{H}_{2} \mathrm{~S}$ action on the TRPV1 receptor have to be determined in future experiments.

In summary, our data suggest that $\mathrm{H}_{2} \mathrm{~S}$ induces pro-nociceptive firing in the peripheral part of the TG nerve through activation of TRPV1 and TRPA1 receptors. This is consistent with the ability of $\mathrm{H}_{2} \mathrm{~S}$ to induce membrane currents and $\mathrm{Ca}^{2+}$ transients in cell bodies of TG neurons, which were mediated by TRPV1 and TRPA1 receptors. The endogenous $\mathrm{H}_{2} \mathrm{~S}$ producing enzyme CBS is abundantly expressed in rat TG neurons (Feng et al., 2013) and it is known that inflammation upregulates CBS expression in TG neurons at both protein and mRNA levels (Miao et al., 2014). A similar increase of CBS expression was observed also in rat DRG neurons after streptozotocin (Zhang et al., 2013) and complete

\section{REFERENCES}

Abe, K., and Kimura, H. (1996). The possible role of hydrogen sulfide as an endogenous neuromodulator. J. Neurosci. 16, 1066-1071.

Akopian, A. N., Ruparel, N. B., Jeske, N. A., and Hargreaves, K. M. (2007). Transient receptor potential TRPA1 channel desensitization in sensory neurons is agonist dependent and regulated by TRPV1-directed internalization. J. Physiol. 583, 175-193. doi: 10.1113/jphysiol.2007.133231

Andersson, D. A., Gentry, C., and Bevan, S. (2012). TRPA1 has a key role in the somatic pro-nociceptive actions of hydrogen sulfide. PLoS One 7:e46917. doi: 10.1371/journal.pone.0046917

Andersson, D. A., Gentry, C., Moss, S., and Bevan, S. (2008). Transient receptor potential $\mathrm{Al}$ is a sensory receptor for multiple products of oxidative stress. J. Neurosci. 28, 2485-2494. doi: 10.1523/JNEUROSCI.536907.2008

Benemei, S., De Cesaris, F., Fusi, C., Rossi, E., Lupi, C., and Geppetti, P. (2013). TRPA1 and other TRP channels in migraine. J. Headache Pain 14:71. doi: 10.1186/1129-2377-14-71

Bhatia, M., Zhi, L., Zhang, H., Ng, S.-W., and Moore, P. K. (2006). Role of substance $\mathrm{P}$ in hydrogen sulfide-induced pulmonary inflammation in mice. Am. J. Physiol. Lung Cell. Mol. Physiol. 291, L896-L904. doi: 10.1152/ajplung. 00053.2006

Deleon, E. R., Stoy, G. F., and Olson, K. R. (2012). Passive loss of hydrogen sulfide in biological experiments. Anal. Biochem. 421, 203-207. doi: 10.1016/j.ab.2011. 10.016

Distrutti, E., Sediari, L., Mencarelli, A., Renga, B., Orlandi, S., Antonelli, E., et al. (2006). Evidence that hydrogen sulfide exerts antinociceptive effects in the gastrointestinal tract by activating KATP channels. J. Pharmacol. Exp. Ther. 316, 325-335. doi: 10.1124/jpet.105.091595

Feng, X., Zhou, Y.-L., Meng, X., Qi, F.-H., Chen, W., Jiang, X., et al. (2013). Hydrogen sulfide increases excitability through suppression of sustained potassium channel currents of rat trigeminal ganglion neurons. Mol. Pain 9:4. doi: 10.1186/1744-8069-9-4

Gavva, N. R., Klionsky, L., Qu, Y., Shi, L., Tamir, R., Edenson, S., et al. (2004). Molecular determinants of vanilloid sensitivity in TRPV1. J. Biol. Chem. 279, 20283-20295. doi: 10.1074/jbc.M312577200

Gerasimova, E., Lebedeva, J., Yakovlev, A., Zefirov, A., Giniatullin, R. A., and Sitdikova, G. (2015). Mechanisms of hydrogen sulfide (H2S) action on synaptic
Freund adjuvant treatments (Qi et al., 2013). We suggest that up-regulation of CSE or CBS during migraine related neuroinflammation in meninges generates $\mathrm{H}_{2} \mathrm{~S}$ resulting in activation of pro-nociceptive TRPV1 and TRPA1 receptors. Associated release of neuropeptide CGRP from TRPV1/TRPA1 positive peptidergic nerve fibers should further support both neuronal sensitization (Giniatullin et al., 2008) and the long-lasting nociceptive firing underlying migraine pain. Therefore, targeting the CBS- $\mathrm{H}_{2} \mathrm{~S}$-TRP signaling in the trigemino-vascular system might represent a novel therapeutic strategy for alleviation of TG pain.

\section{AUTHOR CONTRIBUTIONS}

KK, AM, AY, GS: experimental work and data acquisition. KK, AM, AY, GS: data analysis and preparation of figures. GS, RG, $\mathrm{AH}$ : study design/interpretation and drafting of manuscript. $\mathrm{KK}$, AM, AY, AH, RG, GS: final approval of manuscript.

\section{ACKNOWLEDGMENTS}

The work was supported by Russian Science Foundation No. 1415-00618.

transmission at the mouse neuromuscular junction. Neuroscience 303, 577-585. doi: 10.1016/j.neuroscience.2015.07.036

Gerasimova, E. V., Yakovleva, O. V., Zefirov, A. L., Sitdikova, G. F., Ze, A. L., and Sitdikova, G. F. (2013). Role of ryanodine receptors in the effects of hydrogen sulfide on transmitter release from the frog motor nerve ending. Bull. Exp. Biol. Med. 155, 11-13. doi: 10.1007/s10517-013-2067-7

Giniatullin, R., Nistri, A., and Fabbretti, E. (2008). Molecular mechanisms of sensitization of pain-transducing $\mathrm{P} 2 \mathrm{X} 3$ receptors by the migraine mediators CGRP and NGF. Mol. Neurobiol. 37, 83-90. doi: 10.1007/s12035-008-8020-5

Greiner, R., Pálinkás, Z., Bäsell, K., Becher, D., Antelmann, H., Nagy, P., et al. (2013). Polysulfides link H2S to protein thiol oxidation. Antioxid. Redox Signal. 19, 1749-1765. doi: 10.1089/ars.2012.5041

Hajna, Z., Sághy, E., Payrits, M., Aubdool, A. A., Szoke, E., Pozsgai, G., et al. (2016). Capsaicin-sensitive sensory nerves mediate the cellular and microvascular effects of $\mathrm{H} 2 \mathrm{~S}$ via TRPA1 receptor activation and neuropeptide release. J. Mol. Neurosci. 60, 157-170. doi: 10.1007/s12031-016-0802-z

Hatakeyama, Y., Takahashi, K., Tominaga, M., Kimura, H., and Ohta, T. (2015). Polysulfide evokes acute pain through the activation of nociceptive TRPA1 in mouse sensory neurons. Mol. Pain 11:24. doi: 10.1186/s12990-015-0023-4

Huang, D., Li, S. Y., Dhaka, A., Story, G. M., and Cao, Y.-Q. Q. (2012). Expression of the transient receptor potential channels TRPV1, TRPA1 and TRPM8 in mouse trigeminal primary afferent neurons innervating the dura. Mol. Pain 8:66. doi: 10.1186/1744-8069-8-66

Jordt, S.-E., and Julius, D. (2002). Molecular basis for species-specific sensitivity to "hot" chili peppers. Cell 108, 421-430. doi: 10.1016/s0092-8674(02)00637-2

Kawabata, A., Ishiki, T., Nagasawa, K., Yoshida, S., Maeda, Y., Takahashi, T., et al. (2007). Hydrogen sulfide as a novel nociceptive messenger. Pain 132, 74-81. doi: 10.1016/j.pain.2007.01.026

Kilic, G., Guerrero-Toro, C., Zakharov, A., Vitale, C., Gubert-Olive, M., Koroleva, K., et al. (2017). Serotonergic mechanisms of trigeminal meningeal nociception: implications for migraine pain. Neuropharmacology 116, 160-173. doi: 10.1016/j.neuropharm.2016.12.024

Kimura, H. (2016). Hydrogen polysulfide (H2Sn) signaling along with hydrogen sulfide (H2S) and nitric oxide (NO). J. Neural Transm. (Vienna) 123 , 1235-1245. doi: 10.1007/s00702-016-1600-Z

Lu, W., Li, J., Gong, L., Xu, X., Han, T., Ye, Y., et al. (2014). H2S modulates duodenal motility in male rats via activating TRPV1 and KATP channels. Br. J. Pharmacol. 171, 1534-1550. doi: 10.1111/bph.12562 
Macpherson, L. J., Dubin, A. E., Evans, M. J., Marr, F., Schultz, P. G., Cravatt, B. F., et al. (2007). Noxious compounds activate TRPA1 ion channels through covalent modification of cysteines. Nature 445, 541-545. doi: 10.1038/nature05544

Masuoka, T., Kudo, M., Yamashita, Y., Yoshida, J., Imaizumi, N., Muramatsu, I., et al. (2017). TRPA1 channels modify TRPV1-mediated current responses in dorsal root ganglion neurons. Front. Physiol. 8:272. doi: 10.3389/fphys.2017. 00272

Matsunami, M., Tarui, T., Mitani, K., Nagasawa, K., Fukushima, O., Okubo, K., et al. (2009). Luminal hydrogen sulfide plays a pronociceptive role in mouse colon. Gut 58, 751-761. doi: 10.1136/gut.2007.144543

Miao, X., Meng, X., Wu, G., Ju, Z., Zhang, H.-H., Hu, S., et al. (2014). Upregulation of cystathionine- $\beta$-synthetase expression contributes to inflammatory pain in rat temporomandibular joint. Mol. Pain 10:9. doi: 10.1186/17448069-10-9

Miyamoto, R., Koike, S., Takano, Y., Shibuya, N., Kimura, Y., Hanaoka, K., et al. (2017). Polysulfides (H2Sn) produced from the interaction of hydrogen sulfide (H2S) and nitric oxide (NO) activate TRPA1 channels. Sci. Rep. 7:45995. doi: $10.1038 /$ srep45995

Miyamoto, R., Otsuguro, K.-I., and Ito, S. (2011). Time- and concentrationdependent activation of TRPAl by hydrogen sulfide in rat DRG neurons. Neurosci. Lett. 499, 137-142. doi: 10.1016/j.neulet.2011.05.057

Mustafina, A. N., Yakovlev, A. V., Gaifullina, A. S., Weiger, T. M., Hermann, A., and Sitdikova, G. F. (2015). Hydrogen sulfide induces hyperpolarization and decreases the exocytosis of secretory granules of rat GH3 pituitary tumor cells. Biochem. Biophys. Res. Commun. 465, 825-831. doi: 10.1016/j.bbrc.2015. 08.095

Ogawa, H., Takahashi, K., Miura, S., Imagawa, T., Saito, S., Tominaga, M., et al. (2012). H2S functions as a nociceptive messenger through transient receptor potential ankyrin 1 (TRPA1) activation. Neuroscience 218, 335-343. doi: 10.1016/j.neuroscience.2012.05.044

Okubo, K., Matsumura, M., Kawaishi, Y., Aoki, Y., Matsunami, M., Okawa, Y., et al. (2012). Hydrogen sulfide-induced mechanical hyperalgesia and allodynia require activation of both Cav3.2 and TRPA1 channels in mice. Br. J. Pharmacol. 166, 1738-1743. doi: 10.1111/j.1476-5381.2012.01886.x

Palazzo, E., Rossi, F., de Novellis, V., and Maione, S. (2013). Endogenous modulators of TRP channels. Curr. Top. Med. Chem. 13, 398-407. doi: 10.2174/1568026611313030014

Patacchini, R., Santicioli, P., Giuliani, S., and Maggi, C. A. (2004). Hydrogen sulfide (H2S) stimulates capsaicin-sensitive primary afferent neurons in the rat urinary bladder. Br. J. Pharmacol. 142, 31-34. doi: 10.1038/sj.bjp.0705764

Pingle, S. C., Matta, J. A., and Ahern, G. P. (2007). Capsaicin receptor: TRPV1 a promiscuous TRP channel. Handb. Exp. Pharmacol. 179, 155-171. doi: 10.1007/978-3-540-34891-7_9

Pozsgai, G., Hajna, Z., Bagoly, T., Boros, M., Kemény, Á., Materazzi, S., et al. (2012). The role of transient receptor potential ankyrin 1 (TRPA1) receptor activation in hydrogen-sulphide-induced CGRP-release and vasodilation. Eur. J. Pharmacol. 689, 56-64. doi: 10.1016/j.ejphar.2012.05.053

Qi, F., Zhou, Y., Xiao, Y., Tao, J., Gu, J., Jiang, X., et al. (2013). Promoter demethylation of cystathionine- $\beta$-synthetase gene contributes to inflammatory pain in rats. Pain 154, 34-45. doi: 10.1016/j.pain.2012.07.031

Qu, R., Tao, J., Wang, Y., Zhou, Y., Wu, G., Xiao, Y., et al. (2013). Neonatal colonic inflammation sensitizes voltage-gated $\mathrm{Na}^{+}$channels via upregulation of cystathionine $\beta$-synthetase expression in rat primary sensory neurons. Am. J. Physiol. Gastrointest. Liver Physiol. 304, G763-G772. doi: 10.1152/ajpgi. 00466.2012

Renga, B. (2011). Hydrogen sulfide generation in mammals: the molecular biology of cystathionine- $\beta$ - synthase (CBS) and cystathionine- $\gamma$-lyase (CSE). Inflamm. Allergy Drug Targets 10, 85-91. doi: 10.2174/187152811794776286

Schicho, R., Krueger, D., Zeller, F., Von Weyhern, C. W. H., Frieling, T., Kimura, H., et al. (2006). Hydrogen sulfide is a novel prosecretory neuromodulator in the guinea-pig and human colon. Gastroenterology 131, 1542-1552. doi: 10.1053/j.gastro.2006.08.035
Shatillo, A., Koroleva, K., Giniatullina, R., Naumenko, N., Slastnikova, A. A., Aliev, R. R., et al. (2013). Cortical spreading depression induces oxidative stress in the trigeminal nociceptive system. Neuroscience 253, 341-349. doi: 10.1016/j. neuroscience.2013.09.002

Sitdikova, G. F., Fuchs, R., Kainz, V., Weiger, T. M., and Hermann, A. (2014). Phosphorylation of BK channels modulates the sensitivity to hydrogen sulfide (H2S). Front. Physiol. 5:431. doi: 10.3389/fphys.2014.00431

Sitdikova, G. F., Khaertdinov, N. N., and Zefirov, A. L. (2011). Role of calcium and potassium channels in effects of hydrogen sulfide on frog myocardial contractility. Bull. Exp. Biol. Med. 151, 163-166. doi: 10.1007/s10517-0111280-5

Sitdikova, G. F., Weiger, T. M., and Hermann, A. (2010). Hydrogen sulfide increases calcium-activated potassium (BK) channel activity of rat pituitary tumor cells. Pflugers Arch. 459, 389-397. doi: 10.1007/s00424-009-0737-0

Storti, B., Di Rienzo, C., Cardarelli, F., Bizzarri, R., and Beltram, F. (2015). Unveiling TRPV1 spatio-temporal organization in live cell membranes. PLoS One 10:e0116900. doi: 10.1371/journal.pone.0116900

Susankova, K., Tousova, K., Vyklicky, L., Teisinger, J., and Vlachova, V. (2006). Reducing and oxidizing agents sensitize heat-activated vanilloid receptor (TRPV1) current. Mol. Pharmacol. 70, 383-394. doi: 10.1124/mol.106. 023069

Tang, G., Wu, L., Liang, W., and Wang, R. (2005). Direct stimulation of K(ATP) channels by exogenous and endogenous hydrogen sulfide in vascular smooth muscle cells. Mol. Pharmacol. 68, 1757-1764. doi: 10.1124/mol.105. 017467

Trevisani, M., Patacchini, R., Nicoletti, P., Gatti, R., Gazzieri, D., Lissi, N., et al. (2005). Hydrogen sulfide causes vanilloid receptor 1-mediated neurogenic inflammation in the airways. Br. J. Pharmacol. 145, 1123-1131. doi: 10.1038/sj. bjp.0706277

Xu, G.-Y., Winston, J. H., Shenoy, M., Zhou, S., Chen, J. D. Z., and Pasricha, P. J. (2009). The endogenous hydrogen sulfide producing enzyme cystathionine- $\beta$ synthase contributes to visceral hypersensitivity in a rat model of irritable bowel syndrome. Mol. Pain 5:44. doi: 10.1186/1744-8069-5-44

Yakovlev, A. V., Kurmasheva, E. D., Giniatullin, R., Khalilov, I., and Sitdikova, G. F. (2017). Hydrogen sulfide inhibits giant depolarizing potentials and abolishes epileptiform activity of neonatal rat hippocampal slices. Neuroscience 340, 153-165. doi: 10.1016/j.neuroscience.2016. 10.051

Zakharov, A., Vitale, C., Kilic, G., Koroleva, K., Fayuk, D., Shelukhina, I., et al. (2015). Hunting for origins of migraine pain: cluster analysis of spontaneous and capsaicin-induced firing in meningeal trigeminal nerve fibers. Front. Cell. Neurosci. 9:287. doi: 10.3389/fncel.2015.00287

Zhang, H.-H., Hu, J., Zhou, Y.-L., Hu, S., Wang, Y.-M., Chen, W., et al. (2013). Promoted interaction of nuclear factor- $\kappa B$ with demethylated cystathionine- $\beta$-synthetase gene contributes to gastric hypersensitivity in diabetic rats. J. Neurosci. 33, 9028-9038. doi: 10.1523/JNEUROSCI.106813.2013

Zhu, L., Zhao, L., Qu, R., Zhu, H.-Y., Wang, Y., Jiang, X., et al. (2015). Adrenergic stimulation sensitizes TRPV1 through upregulation of cystathionine $\beta$ synthetase in a rat model of visceral hypersensitivity. Sci. Rep. 5:16109. doi: $10.1038 /$ srep 16109

Conflict of Interest Statement: The authors declare that the research was conducted in the absence of any commercial or financial relationships that could be construed as a potential conflict of interest.

Copyright (c) 2017 Koroleva, Mustafina, Yakovlev, Hermann, Giniatullin and Sitdikova. This is an open-access article distributed under the terms of the Creative Commons Attribution License (CC BY). The use, distribution or reproduction in other forums is permitted, provided the original author(s) or licensor are credited and that the original publication in this journal is cited, in accordance with accepted academic practice. No use, distribution or reproduction is permitted which does not comply with these terms. 